\title{
COMMENTARY \\ Counter-regulation in the IKK family
}

\author{
Luke A. J. O'NEILL ${ }^{1}$ \\ School of Biochemistry and Immunology, Trinity College Dublin, Dublin, Ireland
}

\begin{abstract}
The human IKK [ $\mathrm{I} \kappa \mathrm{B}$ (inhibitor of NF- $\kappa \mathrm{B}$ ) kinase] family has four members; they are the central kinases of innate immunity. Two members, IKK $\alpha$ and IKK $\beta$, the so-called canonical members, phosphoryate $\mathrm{I} \kappa \mathrm{B} \alpha$, leading to activation of the transcription factor $\mathrm{NF}-\kappa \mathrm{B}$ (nuclear factor $\kappa \mathrm{B}$ ), which controls the expression of many immune and inflammatory genes. The IKK-related proteins TBK-1 (TANK-binding kinase 1) and IKK $\varepsilon$ have a different substrate - IRF3 (interferon regulatory factor 3) which regulates a different set of genes, the products of which include Type I interferons. Toll-like receptors (TLRs) such as the lipopolysaccharide receptor TLR4 or the poly(I:C) receptor TLR3 activate each of the IKKs, but the pro-inflammatory cytokine IL-1 (interleukin 1), which signals in a broadly similar way to the TLRs, has so far been shown to activate only the canonical IKKs. In this issue of the Biochemical Journal, Clark et al. bring new insights into the regulation of IKKs. They demonstrate that IL-1 is in fact able to activate IKK $\varepsilon / \mathrm{TBK}-1$, which occurs via
\end{abstract}

IKK $\alpha / \operatorname{IKK} \beta$. The consequence of this is not IRF3 activation, but a negative feedback effect on IKK $\alpha / \operatorname{IKK} \beta$. This provides us with yet another regulatory feedback loop in a system already replete with control mechanisms. It attests yet again to the importance of keeping these innate immune pathways in check, since if they proceed uncontrolled, inflammatory diseases can occur. Importantly, this study utilized new and specific inhibitors of these kinases, suggesting that the interpretation of any effects the compound might have in vivo may be complex, since for example the inhibition of IKK $\varepsilon / \mathrm{TBK}-1$ might actually have a pro-inflammatory effect.

Key words: inhibitor of NF- $\kappa$ B kinase $\varepsilon(\mathrm{IKK} \varepsilon)$, inhibitor of NF- $\kappa$ B kinase IKK $\alpha($ IKK $\alpha)$, inhibitor of NF- $\kappa$ B kinase IKK $\beta$ (IKK $\beta$ ), signal transduction, TANK-binding kinase 1 (TBK-1), Toll-like receptor (TLR), tumour necrosis factor.
Innate immunity is a highly complex process involving a large number of sensors of microbial products [1]. Some of these sensors such as TLR4 (Toll-like receptor 4) and Nlrp3 can also sense a multitude of factors produced by damaged tissue under circumstances of sterile inflammation [2]. A key output from these sensors is the induction of pro-inflammatory cytokines such as IL-1 (interleukin 1) and TNF (tumour necrosis factor), which then amplify the process by boosting expression of similar sets of genes induced by the sensors themselves. This is because IL-1 and TNF can activate similar transcription factors to the initial sensors, notably the transcription factor $\mathrm{NF}-\kappa \mathrm{B}$ (nuclear factor $\kappa \mathrm{B}$ ), whose targets comprise key proteins involved in inflammation and host defence.

The signalling pathway that leads to NF- $\kappa \mathrm{B}$ activation has been the subject of intense investigation over the past 20 years and involves two key kinases: IKK $\alpha[\mathrm{I} \kappa \mathrm{B}$ (inhibitor of $\mathrm{NF}-\kappa \mathrm{B}$ ) kinase $\alpha$ ] and $\operatorname{IKK} \beta$. These kinases phosphorylate $\mathrm{I} \kappa \mathrm{B} \alpha$, leading to the release of the p65/p50 dimer which can then induce gene expression. A second pathway also occurs however in response to TLR4 or the poly(I:C) receptor TLR3, which leads to the activation of IRF3 (interferon regulatory factor 3 ) and the induction of genes encoding such anti-viral proteins as the Type I interferons. This process also involves IKK-like kinases, namely TBK-1 (TANK-binding kinase 1) and IKK $\varepsilon$, which both directly phosphorylate IRF3 leading to its activation [3]. The major change in gene expression induced by many receptors in innate immunity [notably the TLRs, IL-1 and TNF family members, certain NODlike receptors, C-type lectin receptors and RIG-I-like receptors] involves members of the IKK family [4].
This kinase family appears to be especially efficient at responding to stimulation in conserved signalling pathways to induce key proteins for host defence and inflammation. This process can go wrong however, as occurs in a range of immune and inflammatory diseases where these receptor systems have been implicated [5]. The cause of this could lie in a defect in control mechanisms in these pathways, since many inhibitory processes have been identified to modulate responses and keep signals in check.

The study of Clark et al. in this issue of the Biochemical Journal [6] identifies an unexpected inhibitory mechanism inherent in the IKKs themselves. The authors provide evidence that IKK $\varepsilon / \mathrm{TBK}-1$ can phosphorylate IKK $\alpha / \mathrm{IKK} \beta$ directly and inhibit their activity. They also describe the range of substrates phosphorylated by each kinase: some are shared, others are kinase-specific. The finding was made in an analysis of the effect of novel inhibitors of these IKKs; MRT67207 inhibits IKK $\varepsilon /$ TBK-1, whereas BI605906 inhibits IKK $\beta$. In response to IL-1 stimulation, IKK $\varepsilon / \mathrm{TBK}-1$ was activated but did not lead to activation of IRF3. This indicates that these IKKs are necessary, but not sufficient for this process. The IKK $\beta$ inhibitor BI605906 inhibited this effect however, and it was shown that this was due to the inhibition of the direct phosphorylation of IKK $\varepsilon / \mathrm{TBK}-1$ by IKK $\beta$. When the IKK $\varepsilon /$ TBK-1 inhibitor MRT67207 was tested on IL-1 signalling, enhanced phosphorylation of RelA (a substrate for $\operatorname{IKK} \beta$ ) was observed. This suggested an inhibitory feedback effect and this was demonstrated by Clark et al. [6]. MRT67207 enhanced NF- $\kappa$ B-dependent gene transcription. The mechanism of this enhancement was shown to be via increased

Abbreviations used: I 1 B, inhibitor of NF- $\kappa \mathrm{B}$; IKK, I $\kappa \mathrm{B}$ kinase; IL-1, interleukin 1 ; IRF3, interferon regulatory factor 3 ; NF- $\kappa \mathrm{B}$, nuclear factor $\kappa \mathrm{B}$; TAK-1, transforming growth factor- $\beta$-activated kinase 1 ; TBK-1, TANK-binding kinase 1 ; TANK, TRAF-associated NF- $\kappa$ B activator; TRAF, TNF-receptor-associated factor; TLR, Toll-like receptor; TNF, tumour necrosis factor.

${ }^{1}$ Email laoneill@tcd.ie 
phosphorylation of $\operatorname{IKK} \alpha / \operatorname{IKK} \beta$ and it was then shown that IKK $\varepsilon /$ TBK- 1 could in turn phosphorylate IKK $\alpha / \mathrm{IKK} \beta$ thereby inactivating them. A complex cross-talk mechanism therefore was revealed whereby IL- 1 or TLR stimulation activates IKK $\alpha / \mathrm{IKK} \beta$ which phosphorylate IKK $\varepsilon / \mathrm{TBK}-1$. These then phosphorylate $\mathrm{IKK} \alpha / \mathrm{IKK} \beta$, thus inactivating them.

This phenomenon therefore typifies a negative feedback system whereby activation of IKK $\varepsilon /$ TBK -1 by IKK $\alpha /$ IKK $\beta$ leads to the back-phosphorylation of IKK $\alpha / \mathrm{IKK} \beta$ by IKK $\varepsilon / \mathrm{TBK}-1$, inactivating IKK $\alpha / \mathrm{IKK} \beta$. This could in fact set up an oscillation with each cycle comprising activation and inhibition of IKK $\alpha$ / $\operatorname{IKK} \beta$, and this could contribute to the oscillatory nature of $\mathrm{NF}-\kappa \mathrm{B}$ activation, which is thought to contribute to the control of the output from this signalling pathway [7]. Additional complexity was also revealed using TAK-1 (transforming growth factor- $\beta$-activated kinase 1 )-deficient cells. TAK-1 is a key upstream kinase for IKK $\alpha / \mathrm{IKK} \beta$ and it was shown that IL-1 or TLRs (but not TNF) could still activate IKK $\varepsilon / \mathrm{TBK}-1$ in these cells, suggesting another pathway for activation. The kinase in this pathway was not identified, but termed PKX. This kinase may be a key negative kinase for $\operatorname{IKK} \alpha / \operatorname{IKK} \beta$.

Clark et al. [6] also identified other substrates in this system. Again they made use of the specific inhibitors and found something interesting. Previously, it was thought that the two kinase sub-families operated in separate pathways, acting on separate substrates. IKK $\alpha / \operatorname{IKK} \beta$ had been shown to phosphorylate p105, RelA and $\mathrm{I} \kappa \mathrm{B} \alpha$, whereas IKK $\varepsilon / \mathrm{TBK}-1$ phosphoylate IRF3 (IKK $\varepsilon$ and TBK-1) specifically. The authors demonstate that both kinase sub-families phosphorylate NEMO (NF- $\kappa$ B essential modulator), a regulatory scaffold for the IKK complex. They also phosphorylate TANK (TRAF-associated NF- $\kappa$ B activator), a regulatory component of the $\mathrm{IKK} \varepsilon / \mathrm{TBK}-1$ complex. The consequence of these phosphorylations is not fully clear, but they might act to limit IKK $\alpha / \mathrm{IKK} \beta$ further. As the authors point out, it is interesting that the substrates common to all four IKKs are their regulatory and catalytic subunits, whereas the specific substrates are transcription factors specific to sets of genes. This allows for the specific induction of genes, but also allows for cross-talk onto each pathway as a common regulatory mechanism.

A final interesting feature of the study concerns $\operatorname{IKK} \varepsilon$. This protein was originally identified as a gene that was strongly induced by lipopolysaccharide [8]. The function of IKK $\varepsilon$ was however, not known until it was identified as a kinase for IRF3 [3]. Its precise function in the IRF3 activation process has, however, still not fully ellucidated, since TBK-1 appears to be more important. IKK $\varepsilon$ has also been shown to be involved in the activation of the $\mathrm{p} 52$ subunit of $\mathrm{NF}-\kappa \mathrm{B}$ [9]. Its role as a negative regulator of $\operatorname{IKK} \alpha / \mathrm{IKK} \beta$ is however consistent with its inducibility. Several other inhibitors of TLR signalling, such as A20 and SOCS-1, are also highly inducible providing a mechanism for negative feedback. IKK $\varepsilon$ would still have to be activated by $\mathrm{IKK} \alpha / \mathrm{IKK} \beta$, a further step in the process required for IKK $\varepsilon$ to limit IKK $\alpha / \operatorname{IKK} \beta$. This study therefore adds to our knowledge of the functions of the IKK family. Why would the system be set up in this way? In the case of TLR signalling, it might be beneficial to switch from the induction of NF- $\kappa$ B-dependent genes, which are largely pro-inflammatory, to IRF-3-dependent genes, which may be targeted more to host defence or even to antiinflammatory effects as is apparent for interferon- $\beta$ for example [10]. For IL-1 and TNF, it would simply act as another negative feedback mechanism for limiting NF- $\kappa \mathrm{B}$.

The pharmaceutical industry has been very active in the development of IKK $\alpha / \mathrm{IKK} \beta$ inhibitors. The primary goal here was to develop novel anti-inflammatory agents. So far however, there has been limited reported success. This can be for a whole host of reasons, but lack of efficacy may be one. If the inhibition of IKK $\alpha / \mathrm{IKK} \beta$ leads to a decrease in IKK $\varepsilon / \mathrm{TBK}-1$ activity, this could have unanticipated consequences if the induction of genes encoding proteins such as interferon- $\beta$ were to be limited.

Equally, the inhibition of TBK-1 could lead to a boost in IKK $\alpha / \mathrm{IKK} \beta$ activity which would have a proinflammatory effect. Clearly the rather simplistic view of the relative roles of each IKK sub-family will need to be re-evaluated in the context of this study. The further use of MRT67207 and BI605906 as probes to analyse the function of IKKs both in vitro and in vivo will be an important next step. They could in fact be more useful than the analysis of genetically modified mice. It is perhaps not ideal to completely delete a kinase to determine its function rather than modulate activity, owing to the complex dynamic cross-talk processes such as those revealed in this study.

\section{REFERENCES}

1 Takeuchi, 0. and Akira, S. (2010) Pattern recognition receptors and inflammation. Cell 140, 805-820

2 Bianchi, M. E. (2007) DAMPs, PAMPs and alarmins: we all need to know about danger. J. Leukocyte Biol. 81, 1-5

3 Fitzgerald, K. A., McWhirter, S. M., Faia, K. L., Rowe, D. C., Latz, E., Golenbock, D. T., Coyle, A. J., Liao, S. M. and Maniatis, T. (2003) IKKE and TBK1 are essential components of the IRF3 signaling pathway. Nat. Immunol. 4, 491-496

4 Creagh, E. and O'Neill, L. A. J. (2006) TLRs, NLRs and RLRs: a trinity of pathogen sensors that co-operate in innate immunity. Trends Immunol. 27, 352-357

5 Hennessy, E., Parker, A. and O'Neill, L. A. J. (2010) Targeting Toll-like receptors: emerging therapeutics? Nat. Rev. Drug Discov. 9, 293-307

6 Clark, K., Peggie, M., Plater, L., Sorcek, R. J., Young, E. R. R., Madwed, J. B., Hough, J., Mclver, E. G. and Cohen, P. (2011) Novel cross-talk within the IKK family controls innate immunity. Biochem. J. 434, 93-104

7 Nelson, D. E., Ihekwaba, A. E., Elliott, M., Johnson, J. R., Gibney, C. A., Foreman, B. E., Nelson, G., See, V., Horton, C. A., Spiller, D. G. et al. (2004) Oscillations in NF- $\kappa$ B signaling control the dynamics of gene expression. Science 306, 704-708

8 Shimada, T., Kawai, T., Takeda, K., Matsumoto, M., Inoue, J., Tatsumi, Y., Kanamaru, A. and Akira, S. (1999) IKK-i, a novel lipopolysaccharide-inducible kinase that is related to $I_{\kappa}$ B kinases. Int. Immunol. 11, 1357-1362

9 Wietek, C., Cleaver, C. S., Ludbrook, V., Wilde, J., White, J., Bell, D. J., Lee, M., Dickson, M., Ray, K. P. and O'Neill, L. A. J. (2006) IKK $\varepsilon$ interacts with p52 and promotes transactivation via p65. J. Biol. Chem. 281, 34973-34981

10 Mangini, A. J., Lafyatis, R. and Van Seventer, J. M. (2007) Type I interferons inhibition of inflammatory T helper cell responses in systemic lupus erythematosus. Ann. N.Y. Acad. Sci. 1108, 11-23 